\title{
Comparison by Real-Time Hemodynamic and Cardiac Efficiency Monitoring of Sufentanil-Midazolam and Sevoflurane for Anesthesia Induction in Children Undergoing Cardiac Surgery: A Prospective Randomized Study
}

\author{
Ding Han, ${ }^{1,4 *}$ Ya-Guang Liu, ${ }^{4 *}$ Shou-Dong Pan, ${ }^{1}$ Yi Luo, ${ }^{2}$ Jia Li, ${ }^{3 * *}$ Chuan Ou-Yang ${ }^{1,4 * *}$ \\ Departments of ${ }^{1}$ Anesthesia, ${ }^{2}$ Cardiac Surgery, and ${ }^{3}$ Clinical Physiology Laboratory, Capital Institute of Pediatrics Affiliated \\ Children's Hospital, Beijing; ${ }^{4}$ Anesthesia Center, Capital Medical University Affiliated Beijing Anzhen Hospital, Beijing, China
}

\section{ABSTRACT}

Background: Intravenous sufentanil-midazolam and inhalational sevoflurane are widely used for anesthetic induction in children undergoing cardiac surgery. However, knowledge about their effects on hemodynamics and cardiac efficiency remains limited due largely to the lack of direct monitoring method. We used a minimally invasive technique, the pressure recording analytical method (PRAM), to directly monitor hemodynamics and cardiac efficiency, and compared the effects of the two anesthetic regimens in children undergoing ventricular septal defect repair.

Methods: Forty-four children $(2.3 \pm 0.9$ years $)$ were randomized into two groups to receive either intravenous sufentanil $(1 \mu \mathrm{g} / \mathrm{kg})$ and midazolam $(0.2 \mathrm{mg} / \mathrm{kg})$ (Group SM) or 2.0 MAC sevoflurane (Group S) to complete induction after sedation was obtained with 2.0 MAC sevoflurane. Systemic hemodynamic data recorded by PRAM included heart rate (HR), systolic (SBP) and mean (MBP) blood pressure, stroke volume index (SVI), cardiac index (CI), systemic vascular resistance index (SVRI), the maximal slope of systolic upstroke $\left(\mathrm{dp} / \mathrm{dt}_{\max }\right)$ and cardiac cycle efficiency (CCE) after sedation obtained; 1,2 , and 5 minutes after induction achieved; 1, 2, 5, and 10 minutes after intubation.

Results: HR and SVRI showed a decrease in Group SM but an increase in Group S $\left(P_{\text {time }^{*} \text { group }}<0.0001\right)$ in the study period. SVI and CCE showed an increase in Group SM but a decrease in Group S $\left(P_{\text {time* }^{*} \text { roup }}<0.0001\right)$. SBP, MBP, and CI were related to time after polynomial transformation, showing an increase after intubation in Group SM but a decrease in Group S $\left(P_{\text {time }^{2 *} \text { group }}<0.0001\right)$.

Conclusion: PRAM provides meaningful and direct monitoring of hemodynamic parameters as well as cardiac efficiency during the dynamic period of anesthetic induction

Received May 5, 2018; received in revised form October 23, 2018 ; accepted Octobrer 30, 2018.

*Both authors contributed equally to this work; ${ }^{* * B o t h}$ authors contributed equally to this work.

Correspondence: Dr. Chuan Ou-Yang, Capital Medical University Affiliated Beijing Anzhen Hospital, 2 Anzhen Road, Beijing 100029, China; (e-mail: 163-bys@163.com). in children undergoing cardiac surgery. As compared to inhalational sevoflurane, intravenous sufentanil-midazolam exerts more favorable effects on systemic hemodynamics and cardiac efficiency during anesthetic induction in this group of patients.

\section{INTRODUCTION}

Anesthetic induction is challenging in children with congenital heart defect (CHD) undergoing cardiac surgery. The situation may become worse when children are admitted to an operating room without an intravenous access for administration of drugs and fluids. Sevoflurane is feasibly used in weeping and uncooperative children in order to obtain sedation and facilitate venous access [Joshi 2012]. Subsequently, anesthesia induction can be completed by using either intravenous anesthetics or continuous sevoflurane, especially at times of difficult venous catheterization. Intravenous and inhalational anesthetics both induce hemodynamic instability to a certain degree. A large dose of intravenous fentanyl in combination with midazolam was traditionally used to preserve limited cardiac function and attenuate stress response [Gruber 2001; Ikemba 2004]. Later, sufentanil was introduced as an alternative with greater potency in analgesia [Prakanrattana 2002; Xue 2007] and for better maintaining hemodynamic stability [Glenski 1988; Ikeda 1989]. Using sevoflurane alone to complete induction has also been widely used in CHD children [Girotra 1999; Wang 2011; Zeyneloglu 2008]. However, knowledge about their effects on hemodynamics remains limited due largely to the limitations in clinical practice of indirect monitoring parameters, eg heart rate and blood pressures, that do not reflect real hemodynamic status [Sivarajan 2011]. More importantly, both intravenous and inhalational anesthetic agents may induce adverse effects on cardiac efficiency [Coetzee 1989; Hettrick 1996]. A large body of literature has demonstrated that reduced cardiac efficiency is prognostic of adverse outcomes [Nagata 2013; Onorati 2013; Prabhu 2007; Scolletta 2010]. However, little is known about the effects of different anesthetic agents on cardiac efficiency in CHD children.

Echocardiography plays a fundamental role in evaluating cardiac output and cardiac efficiency by means of measuring ventricular-arterial coupling in children [Chen 2001; 


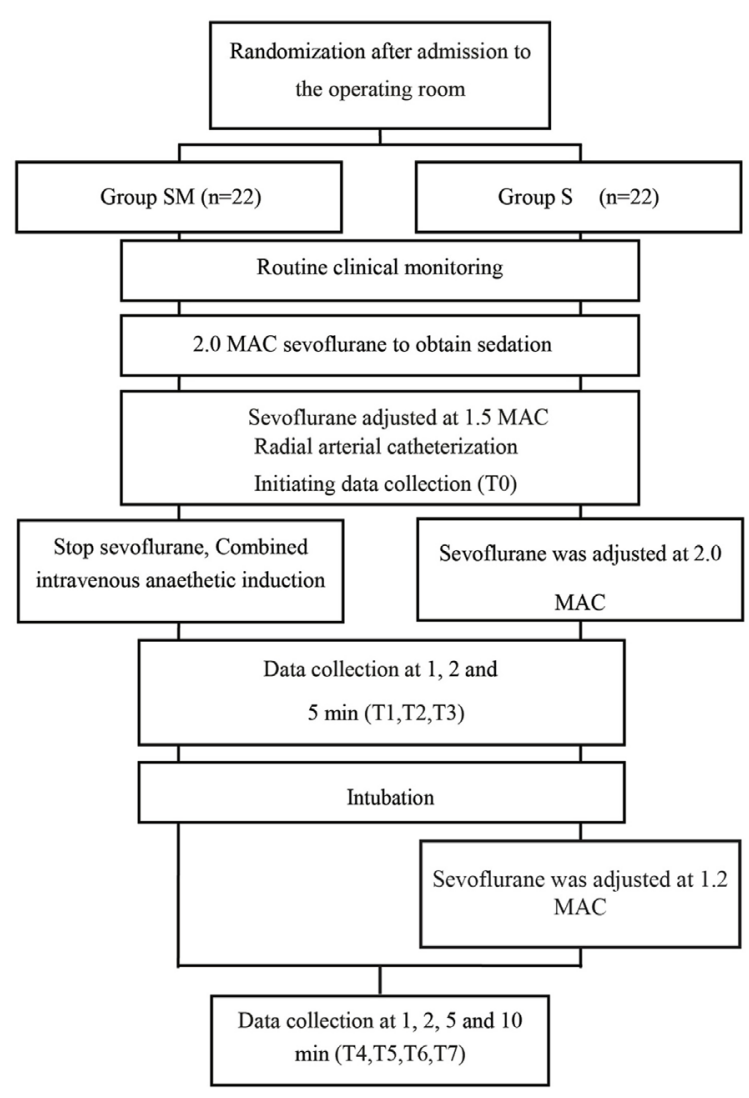

Figure 1. Study protocol.

Khosroshahi 2014; Nagata 2013; Nusmeier 2010]. However, echocardiography assessment is intermittent, operator dependent, and inconvenient in the highly dynamic scenario of the operating room. Efforts have been made to develop user-friendly techniques to directly and continuously assess systemic hemodynamic parameters in highly dynamic clinical settings. Pressure recording analytical method (PRAM) is a minimally invasive, direct, and accurate systemic hemodynamic monitoring technique based on mathematical analysis of the arterial waveform to derive cardiac index (CI), stroke volume index (SVI), systemic vascular resistance index (SVRI), the maximal slope of systolic upstroke $\left(\mathrm{dp} / \mathrm{dt}_{\text {max }}\right.$ ), and cardiac cycle efficiency (CCE) [Romano 2012]. CCE is uniquely derived by PRAM and reflects the ratio of systolic energetic performance to total energetic expenditure of the heart beat and represents the left ventricle wall stress and the heart's effort to maintain an adequate blood flow to tissues. It has been shown to be closely correlated with ventricular-arterial coupling and ventricular ejection fraction in critically ill adults [Scolletta 2013]. A close and negative correlation between CCE and Nt-proBNP has been reported during perioperative period in adults undergoing cardiac surgery [Scolletta 2010].

The present study aimed to compare the effects of intravenous sufentanil-midazolam and inhalational sevoflurane for anesthesia induction on systemic hemodynamics and cardiac efficiency in children undergoing ventricular septal defect repair.

\section{MATERIALS AND METHODS}

\section{Patients}

This study was approved by the Institutional Ethics Committee. Written informed consent was obtained from the parents of all children during the period from November 2016 to September 2017. Eligible patients were older than 1 year, American Society of Anesthesiologists status I or II, without aortic disease (eg aortic valve regurgitation and aortic coarctation), and scheduled for surgical repair of ventricular septal defect using cardiopulmonary bypass. Patients were excluded if they had difficult endotracheal intubation (>2 $\mathrm{min}$ ) or arterial catheter placement (>2 min). Patients investigated by fast flush test that confirmed signal artifacts presence [Scolletta 2013] were also excluded.

\section{Anesthetic Induction Procedure}

After admission to the operating room, children were randomized into one of the two regimens, intravenous sufentanilmidazolam (Group SM) or inhalational sevoflurane (Group S). In both groups, routine clinical monitoring consisted of electrocardiography, pulse oximetry, and capnography. For basal anesthesia, the anesthesia circuit (Datex-Ohmeda Avance, Madison, WI, USA) was primed with $8 \%$ sevoflurane with $100 \%$ oxygen at $6 \mathrm{~L} / \mathrm{min}$ to increase sevoflurane concentration rapidly until age-adjusted 2.0 minimal alveolar concentration (MAC) was achieved. Both groups received sevoflurane via a face mask to obtain sedation. Upon the body movement disappeared, sevoflurane was adjusted to 1.5 MAC and a peripheral intravenous line and a radial arterial catheter were inserted to allow clinical routine monitoring of arterial pressure and the use of PRAM. Subsequently, patients received different agents to complete induction according to group assignment. In Group S, pipecuronium $(0.2 \mathrm{mg} /$ $\mathrm{kg}$ ) was given intramuscularly, and sevoflurane concentration was increased to 2.0 MAC and maintained for 5 mins until intubation. After intubation sevoflurane concentration was maintained at 1.2 MAC to facilitate central venous puncture and catheterization. In Group SM, sevoflurane was terminated, pipecuronium $(0.2 \mathrm{mg} / \mathrm{kg})$, midazolam $(0.2 \mathrm{mg} / \mathrm{kg})$ and sufentanil $(1 \mu \mathrm{g} / \mathrm{kg})$ were given intravenously to complete anesthetic induction, and intubation was performed at 5 mins later. After intubation achieved, mechanical ventilation was initiated with fraction of inspired $\mathrm{O}, 0.5$, tidal volume $10 \mathrm{~mL} /$ $\mathrm{kg}$ and respiratory frequency $15-25$ times/min to maintain end-tidal $\mathrm{CO}_{2}$ partial pressure at $35-40 \mathrm{mmHg}$. After tracheal intubation central venous catheterization manoeuvre (Arrow International, Reading, PA, USA) was applied in both groups.

\section{Direct Monitoring of Systemic Hemodynamic Parameters Using PRAM Design}

PRAM is a minimally invasive hemodynamic monitoring system based on mathematical analysis of the arterial waveform recorded at high sampling rate $(1000 \mathrm{~Hz})$. In any given artery, volume changes occur as a result of the radial expansion in response to variations in pressure. To estimate stroke 

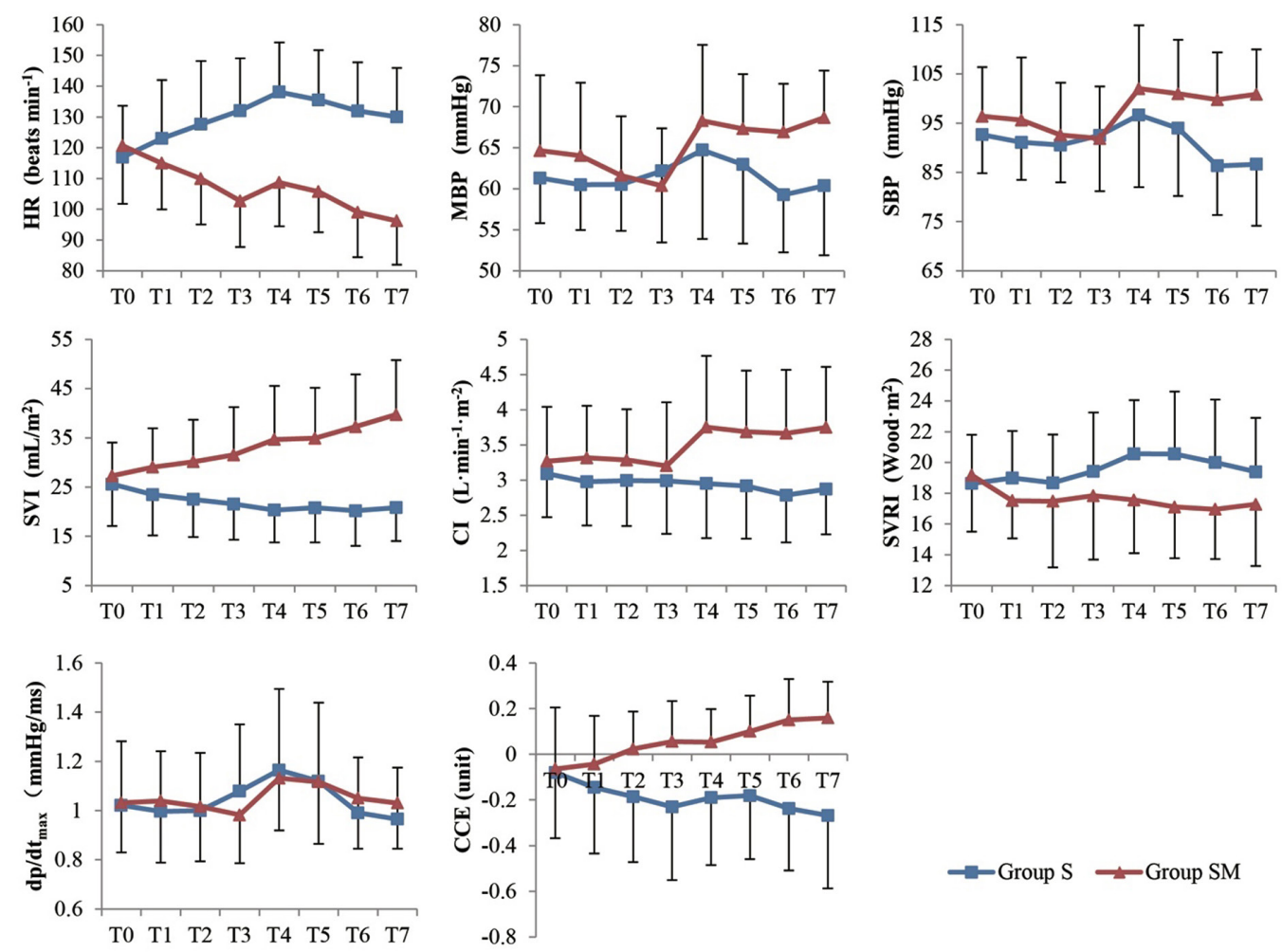

$\rightarrow$ Group S $\rightarrow$ Group SM

Figure 2. The mean $\pm \mathrm{SD}$ values in cardiac cycle efficiency (CCE), cardiac index (Cl), the maximal slope of systolic upstroke $\left(\mathrm{dp} / \mathrm{dt}_{\max }\right)$, heart rate $(\mathrm{HR})$, mean blood pressure (MBP), systolic blood pressure (SBP), stroke volume index (SVI), systemic vascular resistance index (SVRI) in sufentanil-midazolam group (Group SM) and sevoflurane group (Group S) in induction and after intubation. TO: after sedation obtained; T1, T2, T3: 1, 2, 5 min after completion of anesthetic induction, respectively; T4, T5, T6, T7: 1, 2, 5 and $10 \mathrm{~min}$ after intubation, respectively.

volume, the whole instead pulsatile area under the systolic portion of the pressure curve is measured. Simultaneously, arterial impedance $\mathrm{Z}$ is directly determined from both systolic phase and diastolic phase of the pressure waveform, hence no calibration or preloaded data is necessary [Romano 2002]. PRAM provides averaged beat-to-beat calculated data in 30-s intervals and displays data on the screen continuously. Data can be downloaded by dedicated software to Excel spreadsheets for offline analysis. Systemic hemodynamic parameters measured by PRAM included heart rate (HR), systolic (SBP) and mean (MBP) blood pressure, SVI, CI, SVRI, $\mathrm{dp} / \mathrm{dt}_{\max }$, and CCE. Normal range of CCE value is from -1 to +1 unit.

\section{Setup}

A cable is connected between MostCare (core technique PRAM) and a dual output pressure module (Philips, IntelliVue, M8007A) in main monitoring system to obtain signal from the arterial pressure transducer (Edwards Lifesciences Llc, Irvine, CA, USA). As such, MostCare does not have direct connection with the patient.

\section{Study Protocol}

A strict study protocol had been developed (Figure 1). Children were randomized into one of the two induction protocols according to a computer-generated random numbers table. A sealed envelope containing random numbers was opened after patients' arrival to the operating room. The fast flush test was

Table 1. Clinical Data of 44 Children Receiving SufentanilMidazolam (Group SM) or Sevoflurane (Group S) for Anesthesia Induction during Ventricular Septal Defect Repair

\begin{tabular}{lccc}
\hline & $\begin{array}{c}\text { Group SM } \\
(\mathrm{n}=22)\end{array}$ & $\begin{array}{c}\text { Group S } \\
(\mathrm{n}=22)\end{array}$ & $P$ \\
\hline Age, $y$ & $2.0 \pm 0.9$ & $2.6 \pm 1.0$ & .09 \\
Weight, $\mathrm{kg}$ & $11.8 \pm 2.0$ & $13.6 \pm 3.5$ & .06 \\
Height, cm & $88 \pm 8$ & $93 \pm 11$ & .10 \\
Body surface area, $\mathrm{m}^{2}$ & $0.53 \pm 0.07$ & $0.58 \pm 0.11$ & .08 \\
Sex, M/F & $8 / 13$ & $10 / 12$ & .63 \\
ASA, I/II & $14 / 7$ & $12 / 10$ & .42 \\
VSD type, perimembranous/subarterial & $16 / 6$ & $13 / 9$ & .34 \\
MPA-to-AA diameter ratio & $1.43 \pm 0.21$ & $1.53 \pm 0.27$ & .45 \\
& & &
\end{tabular}

ASA indicates American Society of Anesthesiologists status; MPA, main pulmonary artery; AA, ascending aorta. 
Table 2. Mean \pm SD Values and Statistical Results of Systemic Hemodynamics and Cardiac Efficiency in Sufentanil-Midazolam Group (Group SM, $\mathrm{n}=22$ ) and Sevoflurane Group (Group S, $\mathrm{n}=22$ ) during Anesthetic Induction and Intubation in Children Undergoing Ventricular Septal Defect Repair

\begin{tabular}{|c|c|c|c|c|c|c|c|c|c|}
\hline & Group & TO & $\mathrm{T} 1$ & $\mathrm{~T} 2$ & T3 & $\mathrm{T} 4$ & T5 & T6 & $\mathrm{T7}$ \\
\hline \multirow[t]{2}{*}{$\mathrm{HR}$, beats $/ \mathrm{min}^{1}$} & Group SM & $121 \pm 19$ & $115 \pm 15$ & $110 \pm 15$ & $103 \pm 15$ & $109 \pm 14$ & $106 \pm 13$ & $99 \pm 15$ & $96 \pm 14$ \\
\hline & Group S & $117 \pm 17$ & $123 \pm 19$ & $128 \pm 21$ & $132 \pm 17$ & $138 \pm 16$ & $136 \pm 16$ & $132 \pm 16$ & $130 \pm 16$ \\
\hline $\mathrm{SBP}, \mathrm{mmHg}$ & Group S & $93 \pm 8$ & $91 \pm 8$ & $91 \pm 8$ & $93 \pm 11$ & $97 \pm 15$ & $94 \pm 14$ & $86 \pm 10$ & $87 \pm 13$ \\
\hline \multirow[t]{2}{*}{ MBP, $\mathrm{mmHg}$} & Group SM & $65 \pm 9$ & $64 \pm 9$ & $62 \pm 7$ & $60 \pm 7$ & $68 \pm 9$ & $67 \pm 7$ & $67 \pm 6$ & $69 \pm 6$ \\
\hline & Group S & $61 \pm 5$ & $60 \pm 6$ & $61 \pm 6$ & $62 \pm 9$ & $65 \pm 11$ & $63 \pm 10$ & $59 \pm 7$ & $60 \pm 8$ \\
\hline \multirow[t]{2}{*}{ SVI, $\mathrm{mL} \mathrm{m} \mathrm{m}^{-2}$} & Group SM & $27 \pm 7$ & $29 \pm 8$ & $30 \pm 9$ & $32 \pm 10$ & $35 \pm 11$ & $35 \pm 10$ & $37 \pm 11$ & $40 \pm 11$ \\
\hline & Group S & $26 \pm 9$ & $23 \pm 8$ & $23 \pm 8$ & $22 \pm 7$ & $20 \pm 7$ & $21 \pm 7$ & $20 \pm 7$ & $21 \pm 7$ \\
\hline SVRI, Wood m² & Group S & $18.6 \pm 3.2$ & $19 \pm 3.1$ & $18.7 \pm 3.2$ & $19.4 \pm 3.8$ & $20.6 \pm 3.5$ & $20.5 \pm 4.1$ & $20 \pm 4.1$ & $19.4 \pm 3.5$ \\
\hline \multirow[t]{2}{*}{$\mathrm{dp} / \mathrm{dtmax}, \mathrm{mmHg} / \mathrm{ms}^{1}$} & Group SM & $1.03 \pm 0.2$ & $1.04 \pm 0.25$ & $1.02 \pm 0.22$ & $0.98 \pm 0.2$ & $1.13 \pm 0.21$ & $1.12 \pm 0.25$ & $1.05 \pm 0.21$ & $1.03 \pm 0.19$ \\
\hline & Group S & $1.02 \pm 0.26$ & $1 \pm 0.24$ & $1 \pm 0.23$ & $1.08 \pm 0.27$ & $1.16 \pm 0.33$ & $1.12 \pm 0.32$ & $0.99 \pm 0.22$ & $0.97 \pm 0.21$ \\
\hline \multirow[t]{2}{*}{ CCE, unit } & Group SM & $-0.06 \pm 0.27$ & $-0.04 \pm 0.21$ & $0.02 \pm 0.16$ & $0.06 \pm 0.18$ & $0.05 \pm 0.14$ & $0.1 \pm 0.16$ & $0.15 \pm 0.18$ & $0.16 \pm 0.16$ \\
\hline & Group S & $-0.08 \pm 0.29$ & $-0.15 \pm 0.29$ & $-0.19 \pm 0.29$ & $-0.23 \pm 0.32$ & $-0.19 \pm 0.29$ & $-0.18 \pm 0.28$ & $-0.24 \pm 0.27$ & $-0.27 \pm 0.32$ \\
\hline
\end{tabular}

CCE indicates cardiac cycle efficiency; $\mathrm{Cl}$, cardiac index; $\mathrm{dp} / \mathrm{dt}_{\max }$, the maximal slope of systolic upstroke; HR, heart rate; MBP, mean blood pressure; SBP, systolic blood pressure; SVI, stroke volume index; SVRI, systemic vascular resistance index; T0: immediately after sedation and arterial catheterization; T1, T2, T3: 1, 2, 5 min after induction anesthesia, respectively; T4, T5, T6, T7: 1, 2, 5 and 10 min after intubation, respectively. $P_{\text {time }}$ indicates early trend; $P_{\text {time }}$ indicates the following part of trend; $P_{\text {group }}$ indicates the general difference between the two groups; $P_{\text {group time }}$ indicates the difference in early trend between the two groups; $P_{\text {group *time }}{ }^{2}$ indicates the difference in the following part of trend.

employed to preclude the presence of signal artifacts before data collection. Data were collected immediately after sedation obtained (T0), 1, 2, 5 min after completion of anesthetic induction (T1, T2, T3, respectively), and 1, 2, 5, 10 min after intubation (T4, T5, T6, T7, respectively).

\section{Statistical Analysis}

Data are described as mean \pm SD. Student $t$ test and Pearson chi-square test were used to compare the demographic data. Mixed linear regression analysis for repeated measures was used to analyze and compare the changes of the variables in the two groups during the study period. For some measures, polynomial transformation of time was tested regarding the best fit for the time course. The parameter estimates and $\mathrm{P}$ values of time $\left(P_{\text {time }}\right)$ indicate early trend and significance of the change, those of time ${ }^{2}$ $\left(P_{\text {time }^{2}}\right)$ indicate the following trend and significance in the two groups. The parameter estimates and $P$ values of group $\left(P_{\text {group }}\right)$ indicate the significance of the general difference between the groups. The parameter estimates and $P$ values of the interaction of time and group $\left(P_{\text {time }^{*} \text { group }}\right)$ indicate the difference in the early trend of each parameter between the two groups, those of time ${ }^{2}$ and group $\left(P_{\text {time }^{2 *}{ }_{\text {group }}}\right)$ indicate the difference in the following trend of each parameter. All data was performed with SAS statistical software version 9.2 (SAS Institute, Cary, NC, USA). $P<.05$ was considered statistically significant.

\section{RESULTS}

The demographic data of the two groups of patients are shown in Table 1. All patients went through operation uneventfully. None of them had significant adverse events such as cardiac arrest or severe arrhythmia during the study period.

\section{Comparison of the Changes in Hemodynamic Parameters between the Two Groups}

Mean \pm SD values of the hemodynamic parameters are shown in Table 2 and Figure 2. HR showed a decrease 
Table 2 [cont.]

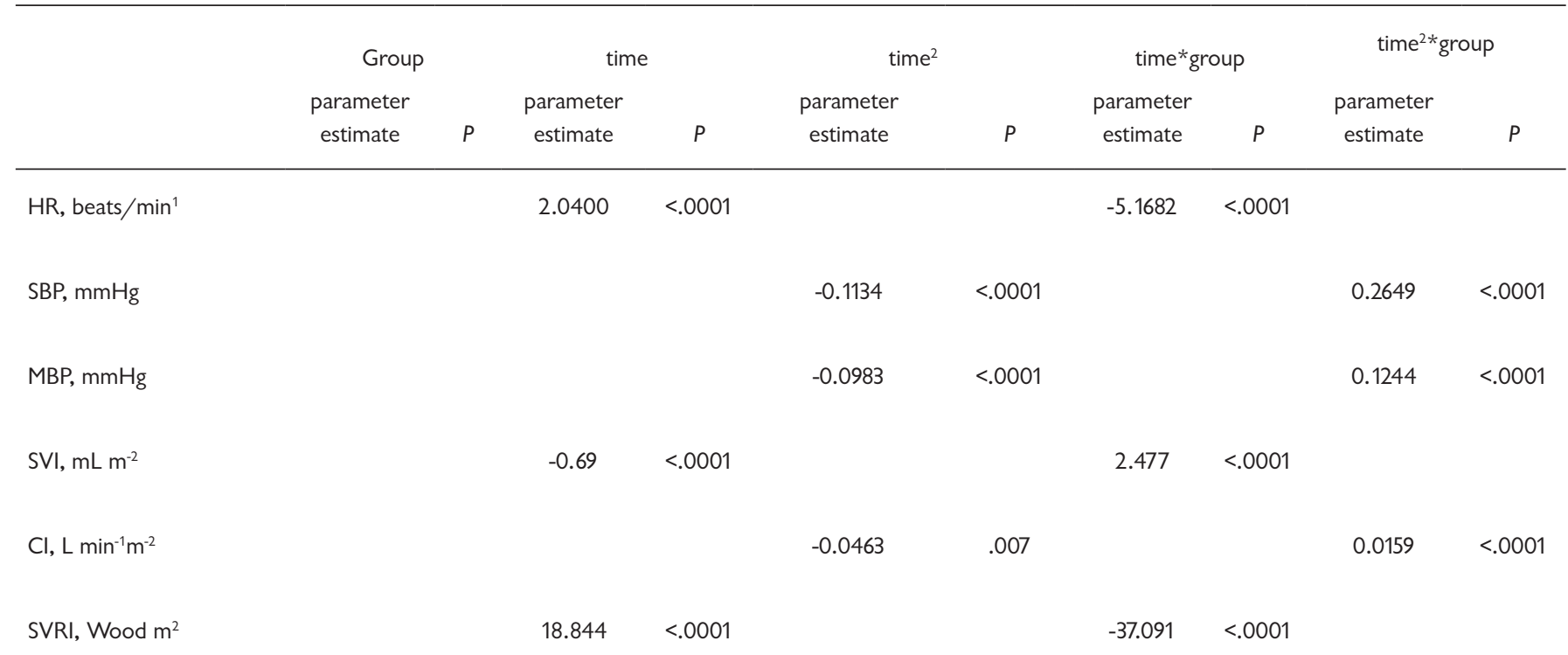

$\mathrm{dp} / \mathrm{dtmax}, \mathrm{mmHg} / \mathrm{ms}^{1}$

in Group SM but an increase in Group S during the study period $\left(P_{\text {timet group }}<.001\right)$. SBP, MBP, and CI were related to time after polynomial transformation, whereby they did not change significantly during T0-T3 in both groups $\left(P_{\text {time }}>\right.$ $.05)$; during T3-T7, they showed an increase in Group SM but a decrease in Group $S\left(P_{\text {time }^{2}}{ }^{*}\right.$ group < .0001). During the study period, SVI and CCE showed an increase in Group SM but a decrease in Group S $\left(P_{\text {timetgroup }}<.0001\right.$ for both). SVRI showed a decrease in Group SM but an increase in Group S $\left(P_{\text {timén }}^{* \text { group }}<.0001\right) . \mathrm{Dp} / \mathrm{dtmax}$ were not related to time and not significantly different between the two groups.

\section{DISCUSSION}

The present study compared directly assessed hemodynamics and cardiac efficiency between two anesthetic induction regimen in children with ventricular septal defect. Our data demonstrated that inhalational regimen maintained less favorable hemodynamics and cardiac efficiency although relatively stable, with significantly higher HR, SVRI, and significantly lower SVI, CI, and CCE, as compared to intravenous regimen.

Cardiovascular stress response to tracheal intubation is one of the noxious stimulus that anesthetists seek to avoid during induction. Our data showed fluctuations in arterials pressure in both groups at intubation, with Group S being more attenuate. This is likely to be ascribed to different levels of anesthesia depth in the two anesthesia induction regimens. Of note, the sevoflurane induction technique in this study is clinical routine and widely used in other studies [Girotra 1999; Wang 2011; Zeyneloglu 2008]. The directly measured hemodynamic parameters by PRAM demonstrated the distinctive and beneficial effects of sufentanil-midazolam regimen on systemic hemodynamics and cardiac efficiency during induction and intubation. Group SM showed a gradual and significant decrease in HR and SVRI, and an increase in SVI and CI, particularly an increase in CCE from -0.06 to +0.16 . The underlying mechanisms for the favorable hemodynamics induced by sufentanil-midazolam regimen over sevoflurane alone are attributed to its effects to reduce SVRI. The supplementation of sedatives to opioid agents has been shown to decrease the stress response more effectively than opioid alone [Lina 1996; Newman 1993], which may contribute to the decrease in SVRI and HR, and consequently an increase in SVI and CI found in our data. Different from our study, a previous [Rivenes 2001] report found that fentanyl-midazolam in combination caused a significant increase in SVR and a decrease in CI secondary to a decrease in HR, whereas sevoflurane maintained cardiac index and heart rate. Different type of opioids may account for the discrepancy. Sufentanil is a synthetic opioid that is five to ten times more potent in analgesia than fentanyl [Prakanrattana 2002; Xue 2007] 
and has the advantage in maintaining hemodynamic stability and relatively low SVRI by means of preserving ventricular function and blocking sympathetic tone [Glenski 1988; Ikeda 1989]. In Group S, sevoflurane regimen caused a significant increase in HR and SVRI, resulting in a significant decrease in SVI and CI. The trend in HR and SVI is in line with a previous report [Ikemba 2004] in infants with functional single ventricle; sevoflurane was associated with significantly increased HR to compensate for the decrease in velocity-time integral (a surrogate for left ventricular stroke volume). The increase in HR secondary to sevoflurane administration was also noted during anesthesia induction in children undergoing non-cardiac surgery [Kern 1997].

General anesthetics interfere with arterial and ventricular mechanical properties, often impairing left ventriculararterial coupling, hence cardiac efficiency. CCE is a unique parameter derived by PRAM as the ratio of systolic energetic performance to total energetic expenditure of the heartbeat. In a cardiac cycle, the energy required to generate a given stoke volume depends on ventricular-arterial coupling. The factors implicated in the arterial system include arterial elastance and reflected waves. The latter are composed of a number of wavelets generated by reflection of previous beats at branchpoints and distal resistive arterioles (backward waves) but also at the aortic valve (forward waves). Depending on their timing according to aortic valve closure and direction, the energy generated by reflected waves can either increase heart work (counted as a negative component in CCE) or facilitate ejection (counted as a positive component in CCE). In our study, CCE showed a continuous increase in Group SM but a continuous decrease in Group S. Dp/ $\mathrm{dt}_{\max }$ were not related to time and not significantly different between the two groups, indicating similar cardiac contractility and performance of the left ventricle. If not by ventricular properties, the improved CCE by sufentanil-midazolam is then attributable to the arterial properties, that is, significantly lower SVRI throughout the study period. The effects of opioids and inhaled agents on cardiac efficiency have been described in adult cardiac surgery after anesthesia induction using Argon wash in technique. Sufentanil groups had higher myocardial efficiency than inhaled agent groups and fentanyl groups [Hoeft 1994]. Sevoflurane has been shown to increase ventricular afterload in a dose-related manner and adversely affect ventricular-arterial coupling in an animal experiment [Deryck 2010], which is in line with our data indicated by SVRI and CCE. Evidence is mounting that CCE might play an important role as a predictor of outcomes [Giglioli 2013; Onorati 2013; Romagnoli 2010; Romano 2006]. Significant improvement in CCE has been frequently observed despite unchanged stoke volume and other standard hemodynamic parameters after cardiovascular surgery [Romagnoli 2010] and interventions [Romano 2006]. A recent report demonstrated that CCE was improved in patients after electrical cardioversion of atrial fibrillation, which was associated with increased SVI and decreased SVRI [Giglioli 2013]. There is a rising call for studies to focus on cardiac energy-based endpoints [Coetzee 1989; Hettrick 1996].

\section{Study Limitations}

There were several limitations in this study. 1) The accuracy of PRAM in CHD children is controversial [AlonsoInigo 2016; Saxena 2013; Urbano 2015]. There have been concerns of some factors that might limit the reliability of PRAM in pediatric patients, such as location of arterial catheter, an over- or under-damping signal from arterial transducer [Urbano 2015]. A recent study [Alonso-Inigo 2016] validated PRAM against the gold standard Fick method in CHD children undergoing cardiac catheterization, and found a good level of agreement in the measurements of cardiac output between the two methods. Additionally, hemodynamic trend tracked by PRAM is meaningful and informative to detect the different hemodynamic effects between the two anesthetic induction regimens. 2) Hemodynamic measurements prior to sedation with sevoflurane were not provided, because it is impossible to place an arterial catheter without sedation in younger children. Therefore, hemodynamic alterations during anesthetic induction were not fully depicted. Nonetheless, baseline hemodynamics were not significantly different between the two groups. 3) The anesthesia depth may not be at the same level in the two anesthesia induction regimens. However, sevoflurane at 2.0 MAC is commonly used for intubation in clinical practice. The concentration of sevoflurane peak at 2.0 MAC was about 5\%-7\% in our study, comparable or lower than that used in similar studies [Girotra 1999; Wang 2011; Zeyneloglu 2008]. Sevoflurane cannot provide satisfactory intubation within 3 minutes [Inomata 1998; Wang 2011]. Even though intramuscular pipecuronium was given, induction with 2.0 MAC sevoflurane was maintained for $\sim 5$ mins to ensure satisfied muscle relaxation in our clinical routine. 4) There is concern that the intracardiac shunting can be substantially altered after inhalation of $100 \%$ oxygen, confusing the hemodynamic effect of anesthetics. Nevertheless, a previous study [Laird 2002] found sevoflurane administered with $100 \%$ oxygen did not change systemic and pulmonary blood flow ratio.

\section{Conclusions}

PRAM provides meaningful and direct monitoring of systemic hemodynamics and cardiac efficiency during the dynamic period of anesthetic induction in children with ventricular septal defect undergoing cardiac surgery. As compared to inhalational sevoflurane, intravenous sufentanilmidazolam exerts significantly more favorable effects on systemic hemodynamics and cardiac efficiency during anesthetic induction. PRAM may be helpful to provide information about the effects of various anesthetic agents on hemodynamics and cardiac efficiency in children with other types of CHD during and after cardiac surgery.

\section{REFERENCES}

Alonso-Inigo JM, Escriba FJ, Carrasco JI, et al. 2016. Measuring cardiac output in children undergoing cardiac catheterization: comparison between the Fick method and PRAM (pressure recording analytical method). Paediatr Anaesth 26:1097-105. 
Chen CH, Fetics B, Nevo E, et al. 2001. Noninvasive single-beat determination of left ventricular end-systolic elastance in humans. J Am Coll Cardiol 38:2028-34.

Coetzee A, Fourie P, Coetzee J, et al. 1989. Effect of various propofol plasma concentrations on regional myocardial contractility and left ventricular afterload. Anesth Analg 69:473-83.

Deryck YL, Fonck K, L DEB, et al. 2010. Differential effects of sevoflurane and propofol anesthesia on left ventricular-arterial coupling in dogs. Acta Anaesthesiol Scand 54:979-86.

Giglioli C, Nesti M, Cecchi E, et al. 2013. Hemodynamic effects in patients with atrial fibrillation submitted to electrical cardioversion. Int J Cardiol 168:4447-50.

Girotra S, Singh A, Mehta Y, et al. 1999. Sevoflurane for induction and intubation in children undergoing congenital cardiac surgery. Ann Card Anaesth 2:27-30.

Glenski JA, Friesen RH, Hassanein RS, et al. 1988. Comparison of the hemodynamic and echocardiographic effects of sufentanil, fentanyl, isoflurane, and halothane for pediatric cardiovascular surgery. J Cardiothorac Anesth 2:147-55.

Hettrick DA, Pagel PS, Warltier DC. 1996. Desflurane, sevoflurane, and isoflurane impair canine left ventricular-arterial coupling and mechanical efficiency. Anesthesiology 85:403-13.

Hoeft A, Sonntag H, Stephan H, et al. 1994. The influence of anesthesia on myocardial oxygen utilization efficiency in patients undergoing coronary bypass surgery. Anesth Analg 78:857-66.

Ikeda K, Ikeda T, Herregods L, et al. 1989. A comparison of hemodynamic changes with sufentanil-O2 and fentanyl-O2 anesthesia for coronary bypass grafting. Masui 38:1469-74.

Ikemba CM, Su JT, Stayer SA, et al. 2004. Myocardial performance index with sevoflurane-pancuronium versus fentanyl-midazolam-pancuronium in infants with a functional single ventricle. Anesthesiology 101:1298-305.

Inomata S, Yamashita S, Toyooka H, et al. 1998. Anaesthetic induction time for tracheal intubation using sevoflurane or halothane in children. Anaesthesia 53:440-5.

Joshi A, Lee S, Pawar D. 2012. An optimum time for intravenous cannulation after induction with sevoflurane in children. Paediatr Anaesth $22: 445-8$.

Kern C, Erb T, Frei FJ. 1997. Haemodynamic responses to sevoflurane compared with halothane during inhalational induction in children. Paediatr Anaesth 7:439-44.

Khosroshahi HE, Ozkan EA, Kilic M. 2014. Arterial and left ventricular end-systolic elastance in normal children. Eur Rev Med Pharmacol Sci 18:3260-6.

Laird TH, Stayer SA, Rivenes SM, et al. 2002. Pulmonary-to-systemic blood flow ratio effects of sevoflurane, isoflurane, halothane, and fentanyl/midazolam with $100 \%$ oxygen in children with congenital heart disease. Anesth Analg 95:1200-6.

Lina AA, Dauchot PJ, Anton AH. 1996. Sufentanil with and without diazepam for coronary artery bypass graft surgery. Acta Anaesthesiol Belg 47:177-85.

Nagata H, Ihara K, Yamamura K, et al. 2013. Left ventricular efficiency after ligation of patent ductus arteriosus for premature infants. J Thorac Cardiovasc Surg 146:1353-8.
Newman M, Reves JG. 1993. Pro: midazolam is the sedative of choice to supplement narcotic anesthesia. J Cardiothorac Vasc Anesth 7:615-9.

Nusmeier A, Van Der Hoeven JG, Lemson J. 2010. Cardiac output monitoring in pediatric patients. Expert Rev Med Devices 7:503-17.

Onorati F, Santini F, Dandale R, et al. 2013. "Polarizing" microplegia improves cardiac cycle efficiency after CABG for unstable angina. Int J Cardiol 167:2739-46.

Prabhu SD. 2007. Altered left ventricular-arterial coupling precedes pump dysfunction in early heart failure. Heart Vessels 22:170-7.

Prakanrattana U, Suksompong S. 2002. Comparison of sufentanil and fentanyl for surgical repair of congenital cardiac defects. J Med Assoc Thai 85 Suppl 3:S807-14.

Rivenes SM, Lewin MB, Stayer SA, et al. 2001. Cardiovascular effects of sevoflurane, isoflurane, halothane, and fentanyl-midazolam in children with congenital heart disease: an echocardiographic study of myocardial contractility and hemodynamics. Anesthesiology 94:223-9.

Romagnoli S, Romano M, Lazzeri C, et al. 2010. Cardiac cycle efficiency: a new index for cardiac work estimation tested during aortic valve plasty. Critical Care 14:P127.

Romano SM, Pistolesi M. 2002. Assessment of cardiac output from systemic arterial pressure in humans. Crit Care Med 30:1834-41.

Romano SM, Olivotto I, Chiostri M, et al. 2006. Minimally Invasive and Noninvasive Hemodynamic Monitoring of the Cardiovascular System: Available Options and Future Perspectives. Current Cardiol Rev 2:37-9.

Romano SM. 2012. Cardiac cycle efficiency: a new parameter able to fully evaluate the dynamic interplay of the cardiovascular system. Int J Cardiol 155:326-7.

Saxena R, Durward A, Puppala NK, et al. 2013. Pressure recording analytical method for measuring cardiac output in critically ill children: a validation study. Br J Anaesth 110:425-31.

Scolletta S, Ranaldi G, Carlucci F, et al. 2010. Relationship between $\mathrm{N}$-terminal pro-B-type natriuretic peptide (Nt-proBNP) and cardiac cycle efficiency in cardiac surgery. Biomed Pharmacother 64:511-5.

Scolletta S, Bodson L, Donadello K, et al. 2013. Assessment of left ventricular function by pulse wave analysis in critically ill patients. Intensive Care Med 39:1025-33.

Sivarajan VB, Bohn D. 2011. Monitoring of standard hemodynamic parameters: heart rate, systemic blood pressure, atrial pressure, pulse oximetry, and end-tidal CO2. Pediatr Crit Care Med 12:S2-11.

Urbano J, Lopez J, Gonzalez R, et al. 2015. Measurement of cardiac output in children by pressure-recording analytical method. Pediatr Cardiol 36:358-64.

Wang KY, Wang HW, Xin LF, et al. 2011. Evaluation of high-concentration sevoflurane for induction and nasotracheal intubation without muscle relaxant for infants with different pulmonary blood flow undergoing surgery for congenital heart diseases. Chin Med J (Engl) 124:4144-8.

Xue FS, Liu KP, Liu Y, et al. 2007. Assessment of small-dose fentanyl and sufentanil blunting the cardiovascular responses to laryngoscopy and intubation in children. Paediatr Anaesth 17:568-74.

Zeyneloglu P, Donmez A, Sener M. 2008. Sevoflurane induction in cyanotic and acyanotic children with congenital heart disease. Adv Ther 25:1-8. 